$$
\begin{aligned}
& \text { GSI PRE } 94.12 \\
& \text { SW } 9413
\end{aligned}
$$
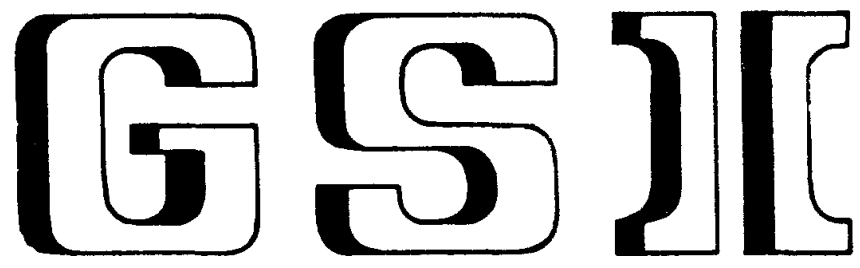

GSI-94-12 PREPRINT

FEBRUAR 1994

TRANSVERSE ENERGY DEPENDENCE OF NEUTRON SQUEEZE-OUT IN RELATIVISTIC HEAVY ION COLLISIONS

S.A. BASS, C. HARTNACK, H. STÖCKER, W. GREINER

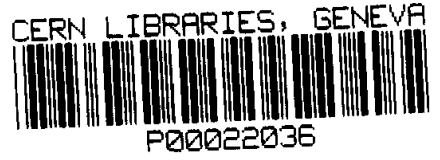

Gesellschaft für Schwerionenforschung $\mathrm{mbH}$ Postfach 110552 - D-64220 Darmstadt · Germany 



\title{
Transverse energy dependence of neutron squeeze-out in relativistic heavy ion collisions*
}

\author{
S. A. Bass ${ }^{a, b}$, C. Hartnack ${ }^{b, c}$, H. Stöcker ${ }^{a}$ and W. Greiner ${ }^{a}$ \\ ${ }^{a}$ Institut für Theoretische Physik der J. W. Goethe Universität \\ Postfach 1119 32, 60054 Frankfurt am Main, Germany \\ ${ }^{b}$ GSI Darmstadt, Postfach 1105 52, 64220 Darmstadt, Germany \\ 'Laboratoire de Physique Nucléaire, Nantes, France
}

February 14, 1994

\begin{abstract}
We present a microscopic calculation of neutron squeeze-out in relativistic heavy ion collisions at beam energies betweeen 400 and $1000 \mathrm{MeV} /$ nucleon. The squeezeout ratio increases monotonously with the transverse momentum of the neutrons. This ratio is independent of the incident beam energy if plotted versus $p_{t} / p_{\text {proj. }}$. We observe a strong dependence on the nuclear equation of state and momentum dependent interaction. An energy dependence manifests itself when plotting the squeeze-out ratio versus the impact parameter.
\end{abstract}

* supported by GSI, BMFT and DFG 
The study of relativistic heavy ion collisions is one of the major tools to investigate the properties of hot and dense nuclear matter $[1,2,3,4,5,6,7,8]$. Among the goals of this research is the determination of the nuclear equation of state and the in-medium properties of nuclear matter. Apart from particle production the study of nuclear collective flow is one of the most promising approaches for investigating those questions. Collective flow effects for nucleons are well established: They have been predicted by nuclear fluid dynamics $[9,10,11,12,13]$ and have experimentally been confirmed at the BEVALAC $[14,15,16]$ for charged particles by the Plastic-Ball collaboration. Microscopic models such as MDM (Molecular Dynamics Model), VUU (Vlasov Uehling Uhlenbeck), and QMD (Quantum Molecular Dynamics) have reproduced the NFD (Nuclear Fluid Dynamics) predictions and show good agreement with the experimental findings $[17,18,19,20,21,22,23,24]$. One has to distinguish between two different types of collective flow: The bounce-off [11] of cold spectator matter from hot and compressed participant matter in the reaction plane and the squeeze-out [12] of this hot and compressed participant matter out of the reaction plane. The most strongly stopped, compressed matter at midrapidity is responsible for the squeeze-out [25]. A strong dependence on the nuclear equation of state for this collective effect is seen $[23,24]$.

The recent measurements of the pion squeeze-out [26] by the KaoS [27] and TAPS [28] collaborations as well as the neutron squeeze-out by the TAPS [28] and LAND [29] collaborations have triggered new interest into this collective effect. In this letter we present the first microscopic calculation of neutron squeeze-out and its energy dependence. We explicitely take the isospin degree of freedom into account.

For our investigation we have calculated samples of $10000 \mathrm{Au}+\mathrm{Au}$ collisions for each of the incident beam energies of $400,600,800$ and $1000 \mathrm{MeV} /$ nucleon, respectively, with the IQMD model which is an extension of the Quantum Molecular Dynamics model (QMD) $[30,31,32,33]$. The IQMD model explicitely incorporates isospin and pion production via the delta resonance $[20,21,34]$. In the QMD model the nucleons are represented by Gaussian shaped density distributions. They are initialized in a sphere of a radius $R=1.12 A^{1 / 3}$ $\mathrm{fm}$, according to the liquid drop model. Each nucleon is supposed to occupy a volume of $h^{3}$, so that the phasespace is uniformly filled. The initial momenta are randomly choosen 
between 0 and the local Thomas-Fermi-momentum. The $A_{P}$ and $A_{T}$ nucleons interact via two- and three-body skyrme forces, a Yukawa potential, momentum dependent interactions, a symmetry potential (to achieve a correct distribution of protons and neutrons in the nucleus) and explicit Coulomb forces between the $Z_{P}$ and $Z_{T}$ protons. Two different equations of state using this ansatz have been implemented: A hard equation of state with a compressibility of $380 \mathrm{MeV}$ and a soft equation of state with a compressibility of 200 $\mathrm{MeV}[35,36]$. For the momentum dependent interaction we use a phenomenological ansatz $[5,31,37]$ which fits experimental measurements $[38,39]$ of the real part of the nucleon optical potential. The nucleons are propagated according to Hamiltons equations of motion. A clear distinction is made between protons and neutrons with coulomb forces acting only on the protons and an asymmetry potential containing the asymmetry term from the Bethe-Weizsäcker formula acting between protons and neutrons. Furthermore parameterized free $p n$ and $p p$ cross sections are used instead of an averaged nucleon-nucleon cross section. They differ by $50 \%$ at $150 \mathrm{MeV}$. Hard $\mathrm{N}$ - $\mathrm{N}$-collisions are included by employing the collision term of the well known VUU/BUU equation [3, 36, 40, 41, 42, 43]. The collisions are done stochastically, in a similar way as in the CASCADE models $[44,45]$. in addition, the Pauli blocking (for the final state) is taken into account by regarding the phase space densities in the final states of a two body collision. Only free unbound protons and neutrons were used for the analysis, all fragments were subtracted using a minimum spanning tree technique.

Figure 1 shows the azimuthal angular distribution of high $p_{t}\left(p_{t} \geq 400 \mathrm{MeV}\right)$ free protons and neutrons at midrapidity. $\varphi$ is the angle between the transverse momentum vector $\overrightarrow{p_{t}}$ and the $x$-axis (which lies in the reaction plane and is perpendicular to the beam axis). The squeeze-out is clearly seen by the pronounced maximum at $\varphi=90^{\circ}$. The full and dashed lines are least square fits with the function $f(\varphi)=a\left(1+s_{1} \cos (\varphi)+s_{2} \cos (2 \varphi)\right)$ which has been used to fit the squeeze-out phenomenon. We use an absolute parameterfree normalization per event. The larger yield for neutrons compared to protons is due to the isospin composition of the gold nuclei. It should be noted, however, that the correct treatment of isospin in inelastic collisions causes a change in the neutron to proton ratio which can be seen in the figure. 
Let us now show the dependence of the observed squeeze-out on increasing beam energy, transverse momentum and impact parameter. We define a squeeze-out ratio [25]

$$
R_{o u t / \text { in }}=\left.\frac{\frac{d N}{d \varphi}\left(\varphi=90^{\circ}\right)+\frac{d N}{d \varphi}\left(\varphi=270^{\circ}\right)}{\frac{d N}{d \varphi}\left(\varphi=0^{\circ}\right)+\frac{d N}{d \varphi}\left(\varphi=180^{\circ}\right)}\right|_{y=y_{C M}}
$$

For values $R_{o u t / \text { in }}>1$ neutrons are emitted preferentially perpendicular to the reaction plane.

Figure 2 shows $R_{\text {out } / \text { in }}$ versus scaled transverse momentum $p_{t} / p_{\text {proj }}$ for the four studied beam energies with cuts on rapidity $\left(-0.15 \leq y_{C M} \leq 0.15\right)$ and impact parameter $(3 \leq$ $b[f m] \leq 9)$. The ratio increases monotonously with the transverse momentum.

This ratio is independent of the the beam energy if the transverse momenta are scaled with the projectile momenta. Therefore, higher transverse momenta must be probed with increasing beam energy if a clean squeeze-out signal is to be isolated. Such a scaling behaviour has already been extracted for the in-plane bounce-off in the hydrodynamic model [46]. It has been experimentally observed in the case of the neutron squeeze-out [47]. Further insight into the reaction mechanism of heavy ion collisions can be gained by this effect, which may serve to predict the magnitude and $p_{t}$ dependence of the neutron squeeze-out for other beam energies than those analyzed in this Letter.

The dependence of the neutron squeeze-out on the momentum dependent interaction and the equation of state for $1 \mathrm{GeV} /$ nucl. is shown in Figure 3: The upper frame shows the hard equation of state with and without momentum dependent interaction (mdi). With mdi, $R_{\text {out } / \text { in }}$ increases by $50 \%$ for large transverse momenta! A difference of $50 \%$ can also be observed by comparing the hard and soft equations of state (both including mdi) in the lower frame of Figure 3: At high transverse momenta the hard equation of state with mdi exhibits a $50 \%$ higher squeeze-out ratio than the soft equation of state with mdi. The hard equation of state without mdi shows a $R_{o u t / \text { in }}$ vs. $p_{t}$ dependence similar to the soft equation of state with mdi. It is important to note, however, that these differences are only seen for high transverse momenta $p_{t} / p_{p r o j} \geq 1$. Analyzing $p_{t}$-integrated data will therefore severely diminish the sensitivity towards the nuclear equation of state and the momentum dependent interaction. 
A cut of $p_{t} / p_{\text {proj }} \geq 0.4$ is employed for the impact parameter dependence of $R_{\text {out } / \text { in }}$ seen Figure 4: The squeeze-out ratio reaches a maximum (within statistical errorbars) for medium impact parameters around $6 \mathrm{fm}$ for $\mathrm{Au}+\mathrm{Au}$ independently of the beam energy in the interval between 400 and $1000 \mathrm{MeV} /$ nucl. Without this cut, the high energies show an increase in $R_{o u t / \text { in }}$ for large impact parameters. However, the squeeze-out ratio exhibits an energy dependence: For $1000 \mathrm{MeV} /$ nucl. and impact parameters up to $8 \mathrm{fm}$ the calculated points consistently lie below those for the lower energies. Figure 4 shows the respective calculations for 400 and $1000 \mathrm{MeV} /$ nucl.. The points for 600 and $800 \mathrm{MeV} /$ nucl. are between the depicted points.

New experimental findings by the TAPS collaboration $[28,48]$ show a neutron squeezeout also at $1 \mathrm{GeV} /$ nucl.. They also exhibit a neutron bounce-off in the reaction plane and present for the first time experimental evidence for the predicted [49] anticorrelation between pions and nucleons in their azimuthal angular distribution for a symmetric system at $1 \mathrm{GeV} /$ nucl..

A direct comparison with experimental results $[14,15,16,28,29]$ must employ the full detector acceptance cuts on the calculation. This can be done for the mature Plastic Ball data (a filter routine is generally accessible [7, 24]). For the fresh data from GSI, such a detailed acceptance routine is not yet publically available. First preliminary comparisons with experimental data [47] at $400 \mathrm{MeV} /$ nucleon look promising.

We have presented a microscopic model dubbed IQMD which incorporates all necessary isospin degrees of freedom. The IQMD model predicts a clear neutron squeeze-out signal. The model exhibits a monotonous $p_{t}$ dependence of the squeeze-out ratio and a broad maximum in the impact parameter dependence around $b=6 \mathrm{fm}$. The squeeze-out ratio versus $p_{t}$ is invariant under the increase in beam energy if scaled with $p_{t} / p_{p r o j}$. However, if the squeeze-out ratio is plotted versus the impact parameter a decrease of the squeeze-out ratio with increasing beam energy can be observed. Most importantly, the squeeze-out ratio exhibits a strong sensitivity towards momentum dependent interactions and the nuclear equation of state for high transverse momenta $p_{t} / p_{\text {proj }} \geq 1$. 


\section{References}

[1] L. P. Csernai and J. I. Kapusta, Phys. Reports 131, 225 (1986).

[2] R. Stock, Phys. Reports 135, 261 (1986).

[3] H. Stöcker and W. Greiner, Phys. Reports 137, 277 (1986).

[4] R. B. Clare and D. Strottman, Phys. Reports 141, 179 (1986).

[5] B. Schürmann, W. Zwermann and R. Malfliet, Phys. Reports 147, 3 (1986).

[6] K. H. Kampert, J. Phys. G15, 691 (1989)

[7] H. H. Gutbrod, A. M. Poskanzer and H. G. Ritter, Rep. Prog. Phys. 52, 1267 (1989).

[8] W. Cassing, V. Metag, U. Mosel and K. Niita, Phys. Reports 188, 365 (1986).

[9] W. Scheid, R. Ligensa, and W. Greiner, Phys. Rev. Lett. 21, 1479 (1968).

[10] W. Scheid, H. Müller, and W. Greiner, Phys. Rev. Lett. 32, 741 (1974).

[11] H. Stöcker, J. A. Maruhn and W. Greiner, Phys. Rev Lett. 44, 725 (1980).

[12] H. Stöcker, L. P. Csernai, G. Graebner, G. Buchwald, H. Kruse, R. Y. Cusson, J. A. Maruhn and W. Greiner, Phys. Rev C25, 1873 (1982).

[13] G. Buchwald, G. Graebner, J. Theiß, J. A. Maruhn, W. Greiner, and H. Stöcker. Phys. Rev. Lett. 52, 1594 (1984).

[14] H.-A. Gustafsson, H. H. Gutbrod, B. Kolb, H. Löhner, B. Ludewigt, A. M. Poskanzer, T. Renner, H. Riedesel, H. G. Ritter, A. Warwick, F. Weik, and H. Wieman, Phys. Rev. Lett. 52, 1590 (1984).

[15] K. G. R. Doss, H. A. Gustafsson, H. H. Gutbrod, K. H. Kampert, B. Kolb, H. Löhner, B. Ludewigt, A. M. Poskanzer, H. G. Ritter, H. R. Schmidt, and H. Wieman, Phys. Rev. Lett. 57, 302 (1986). 
[16] H. H. Gutbrod, K. H. Kampert, B. W. Kolb, A. M. Poskanzer, H. G. Ritter, and H. R. Schmidt, Phys. Lett. B216, 267 (1989).

[17] J. J. Molitoris, J. B. Hoffer, H. Kruse and H. Stöcker, Phys. Rev. Lett. 53, 899 (1984).

[18] W. Schmidt, U. Katscher, B. Waldhauser, J. A. Maruhn, H. Stöcker and W. Greiner, Phys. Rev. C47, 2782 (1993).

[19] J. J. Molitoris and H. Stöcker, Phys. Lett. B162, 47 (1985).

[20] C. Hartnack, L. Zhuxia, L. Neise, G. Peilert, A. Rosenhauer, H. Sorge, J. Aichelin, H. Stöcker, and W. Greiner, Nucl. Phys. A495, 303 (1989).

[21] Ch. Hartnack, GSI-Report 93-5 (1993).

[22] S. M. Kiselev, sub. to Nucl. Phys. A.

[23] Ch. Hartnack, M. Berenguer, A. Jahns, A .v. Keitz, R. Mattiello, A. Rosenhauer, J. Schaffner, Th. Schönfeld, H. Sorge, L. Winckelmann, H. Stöcker and W. Greiner, Nucl. Phys. A538, 53c (1992).

[24] Ch. Hartnack, J. Aichelin, H. Stöcker and W. Greiner, Mod. Phys. Lett. in print.

[25] Ch. Hartnack, H. Stöcker and W. Greiner, Proc. of the Nato Adv. Study Inst. on the Nucl. Equation of State (Peñiscola, Spain), Editors W. Greiner and H. Stöcker, Plenum Press (1990).

[26] S. A. Bass, C. Hartnack, H. Stöcker and W. Greiner. Phys. Rev. Lett. 71, 1144 (1993).

[27] The KaoS collaboration, D. Brill et al., Phys. Rev. Lett. 71, 336 (1993).

[28] The TAPS collaboration, L. Venema et al., Pys. Rev. Lett. 71, 835 (1993).

[29] The LAND collaboration, Y. Leifels et al., Phys. Rev. Lett. 71, 963 (1993).

[30] J. Aichelin and H. Stöcker, Phys. Lett. B176, 14 (1986). 
[31] J. Aichelin, A. Rosenhauer, G. Peilert, H. Stöcker, and W. Greiner, Phys. Rev. Lett. 58, 1926 (1987).

[32] G. Peilert, H. Stöcker, A. Rosenhauer, A. Bohnet, J. Aichelin and W. Greiner, Phys. Rev. C39, 1402 (1989).

[33] J. Aichelin, Phys. Reports 202, 233 (1991).

[34] Ch. Hartnack, H. Stöcker, and W. Greiner, In H. Feldmeier, editor, Proc. of the International Workshop on Gross Properties of Nuclei and Nuclear Excitation, XVI, Hirschegg, Kleinwalsertal, Austria (1988).

[35] J. J Molitoris and H. Stöcker, Phys. Rev C32, R346 (1985).

[36] H. Kruse, B. V. Jacak, and H. Stöcker, Phys. Rev. Lett. 54, 289 (1985).

[37] G. F. Bertsch and S. Das Gupta, Phys. Rep. 160, 189 (1988).

[38] L. G. Arnold et al. Phys, Rev, C25, 936 (1982).

[39] G. Passatore. Nucl. Phys. A95, 694 (1967).

[40] J. Aichelin and G. Bertsch, Phys. Rev. C31, 1730 (1985).

[41] G. F. Bertsch, S. Das Gupta and H. Kruse, Phys. Rep. 160, 189 (1988).

[42] G. Wolf, G. Batko, W. Cassing, U. Mosel, K. Niita, and M. Schäfer, Nucl. Phys. A517, $615(1990)$.

[43] B. A. Li, W. Bauer, and G. F. Bertsch, Phys. Rev. C44, 450 (1991).

[44] Y. Yariv and Z. Frankel, Phys. Rev. C20, 2227 (1979).

[45] J. Cugnon, Phys. Rev. C22, 1885 (1980).

[46] A. Bonasera, L. P. Csernai and B. Schürmann, Nucl. Phys. A476, 159 (1988). 
[47] The LAND collaboration Th. Blaich et al., Talk presented at the NASI on Hot and Dense Nuclear Matter, Bodrum, Turkey 1993 and GSI preprint 94-02 (1994).

[48] A. Kugler, V. Wagner, M. Pachr, M. Sumbera, S. Hlavac and R. S. Simon, Talk presented at the XXIII Mazurian Lakes Summer School on Nucl. Physics, Piaski, Poland, Aug. 18-28, 1993 and GSI preprint GSI-93-75 (1993).

[49] S. A. Bass, C. Hartnack, R. Mattiello, H. Stöcker and W. Greiner, Phys. Lett. B302, $381(1993)$. 


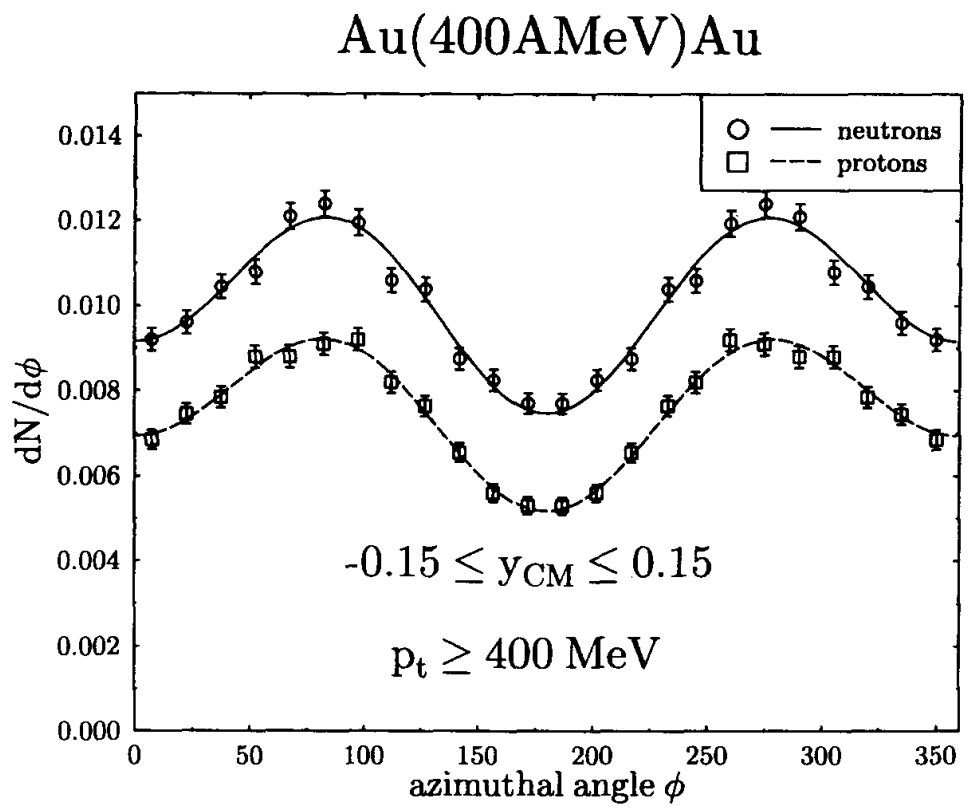

Figure 1: Azimuthal angular distribution for high $p_{t}$ neutrons and protons in $\mathrm{Au}+\mathrm{Au}$ collisions with $400 \mathrm{MeV} /$ nucleon at midrapidity. Only collisions with impact parameters in the range of $3 \leq b \leq 9 \mathrm{fm}$ selected from a minimum bias calculation were used. Protons as well as neutrons exhibit a pronounced peak at $\varphi=90^{\circ}$ perpendicular to the event plane. The higher yield for neutrons is due to the isospin decomposition of the system. 


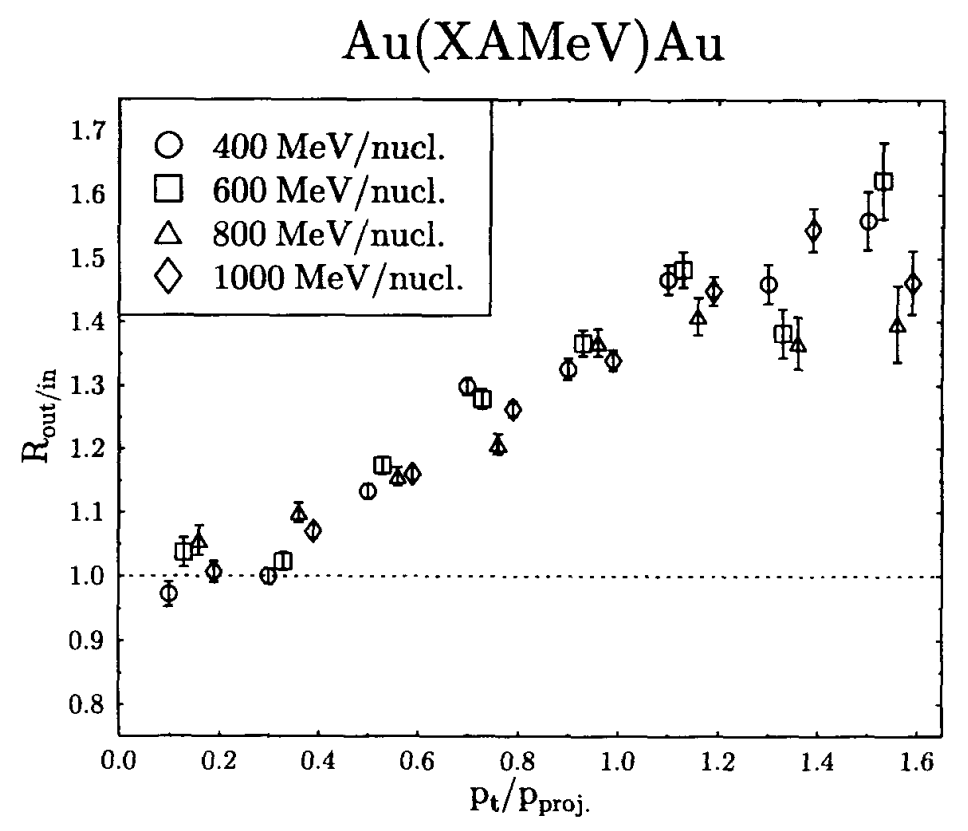

Figure 2: Squeeze-out ratio $R_{\text {out } / \text { in }}$ versus scaled transverse momentum $p_{t} / p_{\text {proj }}$ for neutrons in $\mathrm{Au}+\mathrm{Au}$ collisions at 400,600, 800 and $1000 \mathrm{MeV} /$ nucleon. The ratio increases monotonously with the transverse momentum. By scaling $p_{t}$ with $p_{\text {proj. }}$ the ratio becomes indepent of the incident beam energy. The binning is identical for all 4 energies, the symbols were shifted to increase the readabilty of the figure. 


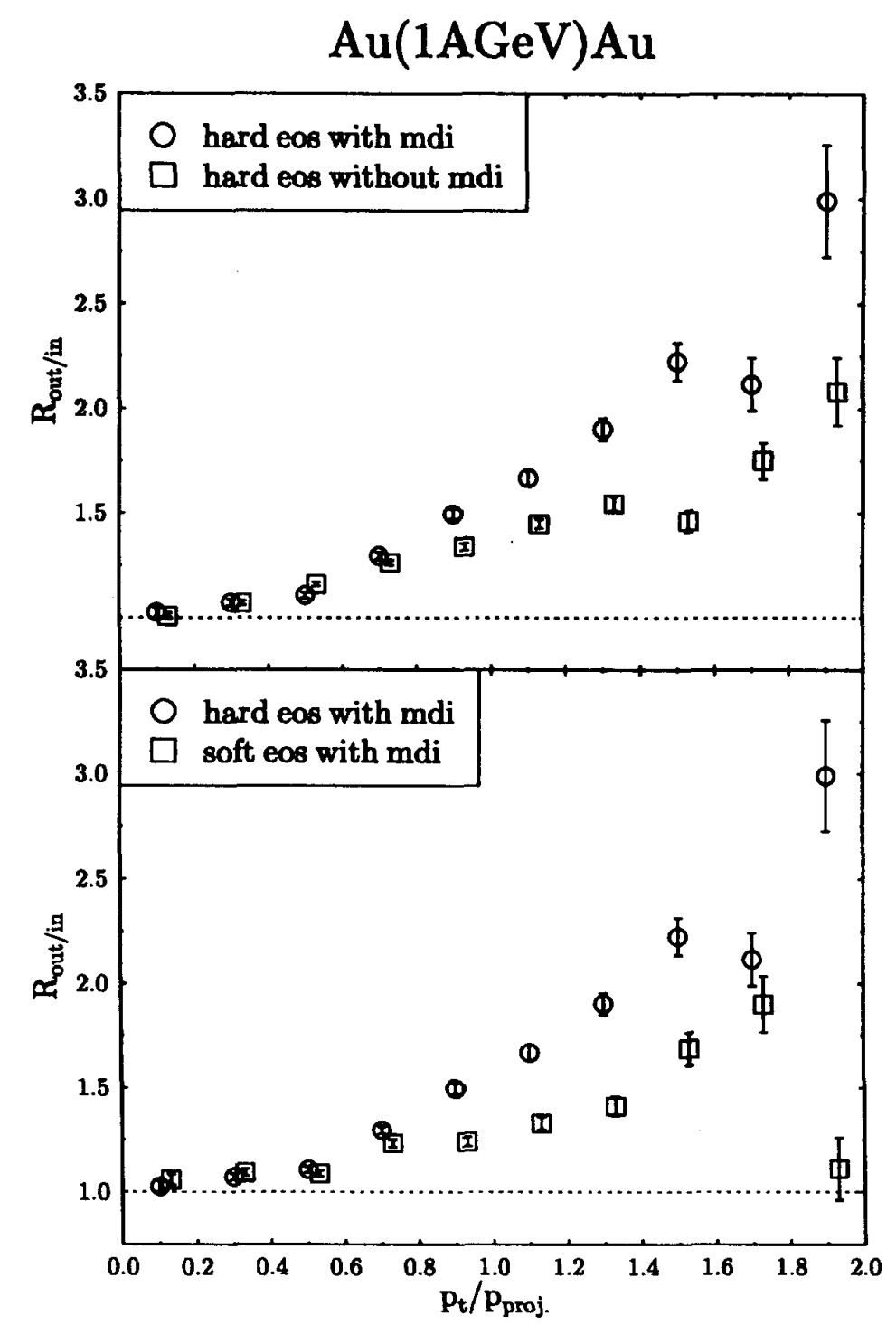

Figure 3: Squeeze-out ratio $R_{\text {out } / \text { in }}$ versus scaled transverse momentum $p_{t} / p_{\text {proj }}$ for neutrons in $\mathrm{Au}+\mathrm{Au}$ collisions at $1 \mathrm{GeV} /$ nucl.. The upper frame shows a comparison between calculations with a hard equation of state with and without momentum dependent interaction (mdi). For large transverse momenta $p_{t} / p_{p r o j} \geq 1$ the calculation with mdi exhibits a $50 \%$ larger squeeze-out ratio. The lower frame shows a comparison between calculations with hard equation of state with mdi and soft equation of state with mdi: Again, for large transverse momenta $p_{t} / p_{\text {proj }} \geq 1$ the hard equation of state shows a $50 \%$ higher squeeze-out ratio than the soft equation of state. 


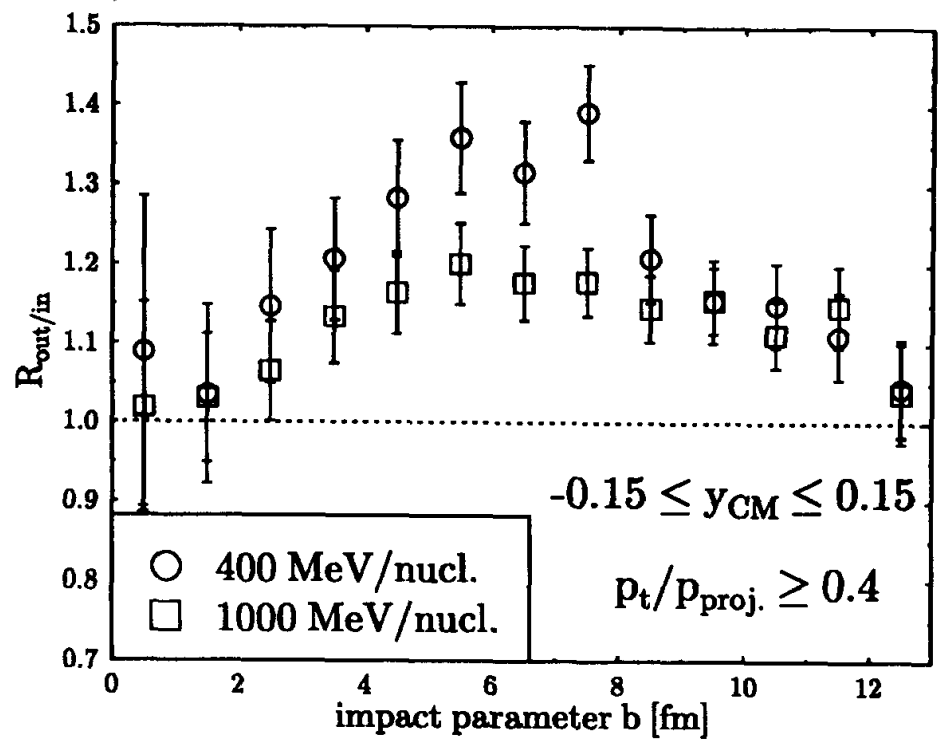

Figure 4: $S$ queeze-out ratio $R_{\text {out } / \text { in }}$ versus impact parameter for high $p_{t}$ neutrons at midrapidity for 400 and $1000 \mathrm{MeV} /$ nucleon. The squeeze-out can best be observed at impact parameters bewteen $3 \mathrm{fm}$ and $9 \mathrm{fm}$. Analogously to figure 2 we employed a cut of $p_{t} / p_{\text {proj. }}$. However, for impact parameters up to $8 \mathrm{fm}$ a diminishing of the squeeze-out with increasing beam energy is visible. The respective curves for 600 and $800 \mathrm{MeV} / \mathrm{nucl}$. lie between those shown but were omitted to increase the readability of the figure. 
$\ldots+\ldots$ 